\title{
Cuando se genera una controversia sociocientífica, ¿aumenta su presencia de noticias en prensa local? El caso del agua en Almería*
}

\section{When a socioscientific issue is generated, does it increase its presence in local press? The case of water in Almería}

\section{Naira Díaz Moreno}

Universidad de Almería

Resumen: En este trabajo estudiamos qué relación existe entre las noticias controvertidas y su presencia en la prensa, examinando el caso concreto del agua en Almería. A través de una metodología variada realizamos el análisis documental, obteniendo una muestra representativa de un periodo normal y otra de un periodo controvertido que situamos en el periodo de mayor difusión de nuestro tópico. Los resultados muestran una escasa presencia para la muestra en estado normal y un abundante promedio diario para el periodo controvertido. Estos resultados y el diferente tratamiento de consensuado o controvertido nos permiten plantear la elección de dos noticias y una alternativa de uso en el aula diferente a las tradicionales propuestas de debates entre partidarios y detractores.

Palabras clave: Controversias sociocientíficas, Alfabetización científica, Prensa.

Abstract: In this study we study the relationship between controversial news and their presence in the press examining specifically the case of water in Almería. Through a varied methodology we carry out the documentary analysis, obtaining a representative sample of a normal period and a controversial sample that we place in the greatest spreading period for our topic. The results show a low presence for the normal sample and an abundant daily average for the controversial sample newspaper. These results and also the different treatment in the news, consensus or controversial, allow us to propose the choice of two news and one alternative use in the classroom, different from the typical tasks based on debates between supporters and opponents to the issue.

Keywords: Socioscientific issues, Scientific Literacy, Press.

(Fecha de recepción: octubre, 2015, y de aceptación: febrero, 2016)

DOI: 10.7203/DCES.31.7036

\footnotetext{
* Parte de este trabajo ha sido posible gracias a la financiación del plan de Formación de Personal Docente e Investigador Predoctoral en Áreas de Conocimiento Deficitarias y del proyecto de excelencia SENSOCIENCIA (P11-SEJ-7385), ambos de la Junta de Andalucía.
} 


\section{Introducción}

La línea de investigación sobre las controversias sociocientíficas nace dentro de la enseñanza de las ciencias como contexto para desarrollar la competencia científica y la alfabetización científica de toda la población. Siguiendo a Couso et al. (2011) y a Pedrinaci et al., (2012) equiparamos competencia científica con alfabetización científica.

Esta competencia o alfabetización científica hace referencia tanto a los conocimientos de los individuos como a su uso para identificar problemas, adquirir otros nuevos, explicar fenómenos científicos y extraer conclusiones basadas en pruebas sobre cuestiones relacionadas con la ciencia. Implica además la comprensión de los rasgos característicos de la ciencia y la percepción del modo en que la ciencia y la tecnología forman parte de nuestro entorno así como la disposición a implicarse en asuntos relacionados con la ciencias y con las ideas de la ciencia como ciudadano reflexivo (OCDE, 2006 citado por Pedrinaci et al., 2012).

Aunque existen distintas propuestas sobre alfabetización o competencia científica todas ellas señalan la necesidad de priorizar en la formación científica no sólo los conocimientos científicos sino también dar entrada a aquellas capacidades que ayuden a la ciudadanía a ejercer sus derechos e integrarse mejor en un mundo cada vez más influido por la ciencia y la tecnología con la finalidad última de tomar decisiones en asuntos relacionados con estos temas.
Así, siguiendo a Blanco (2004) podemos definir esta competencia científica como un conjunto de saberes, de capacidades o de competencias relevantes, entre las que se incluyen leer críticamente la prensa, con la finalidad de comprender y desenvolverse en el mundo actual.

Podemos decir, por tanto, que la competencia o alfabetización científica, considera que la finalidad última de la enseñanza de las ciencias es lograr que toda la población tenga unos conocimientos científicos que le permita no sólo comprender las noticias relacionadas con ciencia y tecnología presentes en los medios de comunicación, sino que además debe tomar decisiones y participar activamente en aspectos relacionadas con temas científico tecnológicos (Domenech, 2014). Con ella, se hace más ambiciosa la educación científica ya que el objetivo es llegar a toda la población y no sólo a los futuros estudiantes de ciencias experimentales.

Si uno de los objetivos del desarrollo de la competencia-alfabetización científica es participar en debates sociocientíficos para la toma de decisiones, las noticias sobre controversias sociocientíficas deben tener presencia en la educación científica (Díaz y Jiménez, 2012) y, en particular, la prensa, que es uno de los principales canales de divulgación de noticias científicas y cuyo uso en el aula presenta un amplio abanico de posibilidades para desarrollar la alfabetización científica y adquirir una cultura tecnocientífica (Gadea, Vilches y Gil, 2009). 
Las problemáticas o controversias sociocientíficas surgen en la ciencia que se está creando, en las fronteras científicas (Díaz, 2013) y por tanto, es de esperar, que los medios se hagan eco de ellas, como en el caso de la oveja Dolly o la manipulación de alimentos transgénicos, realizando así su función divulgativa e informando al público sobre los avances de la ciencia. Por tanto, las noticias con cuestiones sociocientíficas se perfilan como una buena estrategia didáctica para promover el desarrollo del pensamiento crítico (Solbes y Torres, 2012; Solbes, 2013a y Solbes, 2013b), la argumentación (ej. Puig, Bravo y Jiménez-Aleixandre, 2012) y la toma de decisiones fundamentada formando a ciudadanos y ciudadanas que se involucren en debates públicos relacionados con la ciencia y la tecnología.

Sin embargo, como señalan Jarman y McClune (2007), los hechos científicos por sí solos no son noticiables sino que éstos se convierten en noticia cuando son elegidos para aparecer en un medio de comunicación (prensa, televisión, radio, internet) en función de una serie de criterios de "selección de noticias" que marcan los mass media. Estos criterios son los que estos autores denominan como los "valores de la noticia" entre los que destacan el carácter controvertido pues, la existencia de desacuerdo añade dramatismo al hecho relatado, además de emociones fuertes como miedo y suspense.

Este carácter controvertido está latente en numerosos hechos sociocientíficos y solo saltan a la palestra mediá- tica por detonantes determinados. Por ejemplo, el tema del agua en Almería. La población almeriense posee una elevada percepción de déficit hídrico, que convive con una alta demanda de agua por parte de la agricultura, motor de desarrollo económico de la provincia en los últimos años, como así lo ponen de manifiesto la percepción de que el agua es un problema ambiental relevante, detectado en el Ecobarómetro (2004 y 2010). Por tanto, la alta demanda para la agricultura con la percepción de déficit hídrico puede constituir una controversia sociocientífica en Almería como "bien muy valioso", no sólo por su valor intrínseco, su uso diario en las redes de abastecimiento a ciudades a través del agua corriente o el alcantarillado, etc. sino que la gestión urbanística descontrolada en toda la costa mediterránea ha dejado de ser un motor de desarrollo para convertirse en un problema a resolver a nivel de gasto de agua. Sin embargo, el detonante para su mayor presencia en la prensa se produce tras la implantación de la Directiva europea Marco Agua en el 2000 y, sobre todo, con el debate creado en 2004 con la derogación del Plan Hidrológico Nacional y el trasvase del Ebro.

Por ello, en esta publicación, que forma parte de un trabajo más amplio, queremos analizar si el tema del agua, siempre controvertido en Almería, tiene presencia habitual en la prensa local o, si por el contrario, esta presencia aumenta en los periodos detonados por algún hecho que genere mayor controversia como fue el caso del debate sobre el Plan Hidrológico Nacional de 2004. 
Así pues nos planteamos qué relación existe entre las noticias controvertidas y la presencia de estas en prensa: si aparecen, cuánto aparecen estas temáticas controvertidas, dónde existen diferentes puntos de vista y qué repercusiones sociales tienen preguntándonos, ¿cuál es la presencia en prensa local en función de que esté en periodo normal o en periodo de controversia? En definitiva las respuestas a estas preguntas puede permitirnos concluir si la mayor o menor presencia de un tema en prensa permite determinar que éste constituya una controversia sociocientífica.

De este modo, nuestra finalidad última será la de facilitar la búsqueda de noticias controvertidas en prensa a través de indicadores sencillos, uno de ellos la presencia, de manera que pueda ser útil para el profesorado interesado en utilizar las noticias de controversias sociocientíficas en el aula. En futuros trabajos analizaremos la implementación de propuestas de aula que, por motivos de espacio, no son objeto de este trabajo. En el presente artículo nos centraremos en plantear sugerencias para trabajar estas noticias que pueden ser de utilidad para el profesorado basándonos en la elección de dos de ellas y que constituyen una alternativa de uso a las tradicionales propuestas de debates entre partidarios y detractores.

\section{Antecedentes en el análisis de las noticias científicas en prensa}

En la literatura didáctica hemos encontrado estudios de prensa donde se analiza el contenido de temáticas controvertidas (Bowe et al., 2014; Boyd y Paveglio, 2014; Neresini y Lorenzet, 2014; Poberezshkaya, 2015) así como el carácter de consenso-controversia en noticias científicas de prensa escrita (Dimopoulos y Koulaidis, 2002 y 2003; Pedrós y Martínez, 2000; Pedrós, Martínez y Varo, 2007; Vílchez, 2005 y 2009). Sin embargo, en ninguno de estos estudios se especifica cómo se ha llevado a cabo el análisis de la controversia ni cómo se han detectado, con lo que podemos decir, que existe un vacío respecto a cómo determinar controversias sociocientíficas, justificando así la necesidad de nuestra investigación. Desde el punto de vista de la relevancia, esto tiene importantes repercusiones en la comprensión pública de la ciencia a través de la divulgación y en el contexto escolar, ya que la selección de noticias que muestren controversias sociocientíficas debe ser el paso previo de todo docente que quiera utilizar la prensa en el aula de ciencias (Bowe et al., 2014; Jarman y McClune, 2007).

Hemos revisado los estudios previos existentes de noticias científicas en prensa con el objeto de obtener metodologías de análisis documental para utilizarlas y resultados de análisis de otros contextos que nos sirvan como comparación, por ejemplo, para detectar si la presencia de noticias sociocientíficas sobre agua en la prensa almeriense es o no elevada. En este sentido, destacamos la investigación de Dimopoulos y Koulaidis (2003), cuya metodología de presencia por conteo de noticias y por área impre- 
sa utilizaremos, donde obtuvieron como resultado una presencia de cuatro a cinco noticias relevantes diarias de ciencia y tecnología que suponían un área total impresa entre el 1 y el 3\% en los periódicos de mayor tirada de la prensa nacional griega. Lapetina (2005), utilizando una metodología similar a Dimopoulos y Koulaidis (2003) para la prensa escrita almeriense, obtuvo una presencia promedio de 2,9 noticias científicas diarias sumando las relacionadas con cualquier tópico científico (agricultura, medicina, ambientales, etc.) incluyendo los suplementos habituales (entre ellos, Agricultura 2000 y Agro Almería), que disminuye a un promedio de 1,87 noticias diarias al suprimir estos suplementos.

Ambos trabajos (Dimopoulos y Koulaidis, 2003 y Lapetina, 2005), como resumimos en el cuadro I, obtienen como promedio de presencia entre 1 y $3 \%$ del área impresa de las noticias científicas de todos los ámbitos y temáticas. Por tanto, para que cualquier tópico sociocientífico pueda ser considerado como presente (y no anecdótico en prensa) debe superar el 0,7\% del área impresa total (resultado mínimo del cuadro I).

Estos datos en prensa internacional (Bauer et al., 1995; Dimopoulos y Koulaidis, 2002 y 2003; Metcalfe y Gasgoine, 1995; Pellenchia, 1995) nos animan a utilizar como indicador de presencia el promedio de artículos diarios publicados en prensa escrita, reservando el porcentaje de área impresa (tamaño, presencia de imágenes, etc.) como indicador de la importancia que se les concede a estas noticias en el periódico.

\section{Cuadro I: Resultados de los principales estudios sobre presencia de ciencia y tecnología en prensa}

\begin{tabular}{|l|l|l|l|l|l|l|}
\hline & $\begin{array}{l}\text { Dimopoulos } \\
\text { y Koulaidis } \\
(\mathbf{2 0 0 2 , 2 0 0 3 )}\end{array}$ & $\begin{array}{c}\text { Lapetina } \\
\mathbf{( 2 0 0 5 )}\end{array}$ & $\begin{array}{l}\text { Jiménez et al. } \\
\mathbf{( 2 0 1 0 )}\end{array}$ & $\begin{array}{l}\text { Bauer et } \\
\text { al. (1995) }\end{array}$ & $\begin{array}{l}\text { Pellenchia } \\
\mathbf{( 1 9 9 7 )}\end{array}$ & $\begin{array}{l}\text { Metcalfe y } \\
\text { Gasgoine } \\
(\mathbf{1 9 9 5})\end{array}$ \\
\hline $\begin{array}{l}\text { Muestra, } \\
\text { tipología de } \\
\text { la prensa } \\
\text { analizada }\end{array}$ & $\begin{array}{l}4-5 \text { noticias } \\
\text { científicas } \\
\text { diarias en } \\
\text { prensa de tirada } \\
\text { nacional griega }\end{array}$ & $\begin{array}{l}\text { Promedio de } \\
2,9 \text { noticias } \\
\text { científicas } \\
\text { diarias en } \\
\text { prensa de tirada } \\
\text { provincial } \\
\text { almeriense }(1,87 \\
\text { sin suplementos) }\end{array}$ & $\begin{array}{l}43 \text { noticias } \\
\text { sobre energía } \\
\text { solar en prensa } \\
\text { almeriense/61 } \\
\text { días (promedio } \\
0,7)\end{array}$ & $\begin{array}{l}\text { Prensa } \\
\text { nacional } \\
\text { británica }\end{array}$ & $\begin{array}{l}\text { Prensa } \\
\text { nacional } \\
\text { americana }\end{array}$ & $\begin{array}{l}\text { Prensa } \\
\text { nacional } \\
\text { australiana }\end{array}$ \\
\hline $\begin{array}{l}\text { \% área } \\
\text { impresa de } \\
\begin{array}{l}\text { CyT respecto } \\
\text { al total del } \\
\text { periódico }\end{array}\end{array}$ & $1-3 \%$ & $2,3 \%$ & -- & $5 \%$ & $2 \%$ & $2,9 \%$ \\
\hline
\end{tabular}




\section{Metodología}

Como indicábamos en la introducción, la finalidad última de nuestra investigación será facilitar al profesorado la búsqueda de controversias sociocientíficas en prensa escrita para poder utilizar noticias en el aula de ciencias. Para determinar si la presencia en los medios de comunicación puede ser un indicador de que esa noticia científica sea o no controvertida, nos parece necesario comparar la presencia de noticias en un período controvertido con respecto a otro período no controvertido. De manera que si existen diferencias significativas podamos concluir que a mayor controversia, mayor presencia en prensa de noticias relacionadas. De esta forma, la mayor presencia permitiría indicar al profesorado de que se encuentra ante una controversia permitiéndole llevar al aula noticias que realmente han aparecido en prensa durante el período que la temática ha constituido una controversia y otras de la misma temática en un período "normal" y con ello ampliar las típicas actividades de utilizar noticias publicadas en periódicos de distinta línea editorial o con posiciones encontradas (Díaz y Jiménez, 2012) para trabajar las controversias.

Para lograrlo, necesitamos por tanto situar las controversias a lo largo del tiempo para determinar estos dos períodos, normal y controvertido.

Después de realizar una revisión de los estudios realizados sobre consenso-controversia y análisis de noti- cias científicas (Díaz, 2013) tomamos como metodología dos formas distintas y complementarias de muestreo o búsqueda de noticias: "constructed week" y "noticia testigo", que desarrollaremos en este apartado, aplicando ambas al tema del agua en Almería que nos servirán para seleccionar artículos de prensa y poder medir la presencia o promedio de artículos diarios de temas controvertidos, en concreto el tema del agua en la prensa almeriense.

Para la búsqueda de noticias en la prensa almeriense hemos tomado como periódicos de referencia locales aquellos con mayor tirada provincial: La Voz de Almería, con un promedio de tirada de 11549 y 8596 ejemplares diarios para el 2012 y 2011 respectivamente y el diario Ideal (Edición Almería), con una tirada promedio de 3301 ejemplares diarios para el 2012 y 3879 para el 2011.

\section{Metodología "constructed week"}

Esta metodología, ya utilizada en investigaciones previas (Dimopoulos y Koulaidis, 2002; Hansen et al. 1998; Lapetina, 2005), consiste en realizar una cata por semanas y otra por años durante un período de tiempo y nos permite obtener una muestra extensa en el tiempo sin tener una muestra excesiva de artículos. En nuestra investigación hemos tomado semanas completas para evitar comparaciones diarias. Así, se ha tomado una muestra de la prensa local realizando una cata por semanas y otras por años que nos aportará información sobre la presencia de noticias 
relacionadas con el agua entre los años 1992 y 2008 . Hemos seleccionado este período comprendido desde 1992 a 2008 puesto que se corresponde con el desarrollo que experimentó la provincia a nivel económico y cultural durante los años 90 , prolongando el periodo de análisis hasta el 2008 para, en catas cuatrianuales, poder acercarlo lo más posible a la actualidad (al comienzo de este estudio).

Además, durante este tiempo, tuvieron lugar hechos relevantes relacionados con el tema del agua como la derogación del PHN en el 2004, implantación de la DMA en el 2000, etc., lo cual nos llevó a pensar que podía ser significativa de la presencia de noticias relacionadas con el tema del agua.

Por todo ello, para comenzar el análisis de la presencia de noticias hemos tomado los artículos encontrados relacionados con el tópico agua en las últimas semanas completas de los meses de febrero, junio y octubre (representantes de cada trimestre) de los años 1992, 1996, 2000, 2004 y 2008. Seleccionamos por tanto, 15 semanas (105 días) en los que analizamos 98 ejemplares de periódico, estudiando un período anual amplio y obteniendo una muestra representativa de un período "normal" y que además nos permite comparar la presencia de una noticia con respecto a otras en el mismo período de tiempo al estudiar semanas completas.

$\mathrm{Al}$ realizar este estudio con la metodología "constructed week" en la prensa almeriense encontramos 221 artículos relacionados con la ciencia y la tecnología, de los cuales 50 están directamente relacionados con el tema del agua. Estas 50 noticias encontradas constituirán nuestra muestra en estado normal, no excitado o no controvertido. Mostramos algunos de los titulares más relevantes de esta muestra en el cuadro II.

Con la finalidad de completar el estudio de la presencia de noticias y localizar el periodo controvertido hemos analizado la evolución en el tiempo de las noticias utilizando la metodología "noticia testigo" (Fernández-Muerza, 2004) que desarrollamos a continuación.

\section{Metodología "noticia testigo"}

Con esta metodología que realiza búsquedas por temáticas nos centramos, a modo de zoom, en la búsqueda del periodo de mayor difusión de noticias sobre el tema del agua en prensa nacional que nos permita seleccionar el mayor pico de presencia para después, una vez encontrado este período de máxima presencia, repetir el análisis en prensa a nivel local para un mes completo, a lo que llamamos "constructed month".

Los tres diarios nacionales con mayor número de lectores a partir de 2007 (El País, El Mundo y La Vanguardia,), según datos de la Asociación para la Investigación de Medios de Comunicación consultados en su página web www.aimc. es los utilizamos exclusivamente para localizar la mayor presencia de noticias sobre el agua. Hemos utilizado los buscadores online de cada diario con las 


\section{Cuadro II: Titulares de las noticias seleccionadas en el periodo normal o no controvertido}

\begin{tabular}{|c|c|c|}
\hline Título del artículo & Fecha & $\begin{array}{l}\text { Nombre del } \\
\text { diario }\end{array}$ \\
\hline $\begin{array}{l}\text { La Plataforma Solar será escenario de unas jornadas sobre } \\
\text { desintoxicación de aguas residuales industriales }\end{array}$ & $22 / 10 / 1992$ & La Voz de Almería \\
\hline El agua de Galasa no se puede beber & $28 / 06 / 1996$ & La Voz de Almería \\
\hline $\begin{array}{l}\text { La "Plataforma Anti-Térmica" organiza una manifestación contra la } \\
\text { desaladora }\end{array}$ & $30 / 06 / 1996$ & La Voz de Almería \\
\hline $\begin{array}{l}\text { Las barriadas de Aguadulce y La Gloria cuentan desde ayer con } \\
\text { agua de calidad en los grifos }\end{array}$ & $25 / 10 / 1996$ & La Voz de Almería \\
\hline $\begin{array}{l}\text { Los agricultores de Níjar trasladaron sus reivindicaciones a las } \\
\text { calles de la capital }\end{array}$ & $26 / 10 / 1996$ & La Voz de Almería \\
\hline En el Poniente se consume el doble de agua que entra & $25 / 02 / 2000$ & $\begin{array}{l}\text { Ideal (Suplemento } \\
\text { Agro - Almería) }\end{array}$ \\
\hline $\begin{array}{l}\text { La nueva ética del agua exige un uso ecológico y coherente de este } \\
\text { recurso }\end{array}$ & $30 / 06 / 2000$ & $\begin{array}{l}\text { Ideal (Suplemento } \\
\text { Agro - Almería) }\end{array}$ \\
\hline $\begin{array}{l}\text { Encargado el proyecto para la realización del plan global de la red } \\
\text { secundaria de regadío }\end{array}$ & $26 / 10 / 2000$ & La Voz de Almería \\
\hline La Plataforma Solar desarrolla dos proyectos de desalación de agua & $29 / 02 / 2004$ & La Voz de Almería \\
\hline $\begin{array}{l}\text { Diputación presenta al Gobierno un plan para asegurar el } \\
\text { abastecimiento de agua. }\end{array}$ & $28 / 10 / 2004$ & La Voz de Almería \\
\hline La eficiente gestión del agua en Almería aterrizará en Zaragoza & $28 / 06 / 2008$ & Ideal \\
\hline $\begin{array}{l}\text { Indignación en el Almanzora por el apoyo político al fin del trasvase } \\
\text { del Tajo en } 2015\end{array}$ & $20 / 10 / 2008$ & Ideal \\
\hline $\begin{array}{l}\text { Almería enseñará a la región de Orán cómo aprovechar mejor el } \\
\text { agua }\end{array}$ & $23 / 02 / 2008$ & La Voz de Almería \\
\hline El Bajo Andarax pide agua depurada con urgencia & $21 / 02 / 2008$ & Ideal \\
\hline Se descubre el uso de lodos de depuradora como fertilizante & $26 / 06 / 2008$ & La Voz de Almería \\
\hline
\end{tabular}

palabras clave "plan hidrológico nacional", "trasvase del Ebro", "depuración" y "desalación" desde 1992 hasta 2011. Así, determinamos los años y, posteriormente, los meses con mayor número de noticias relacionadas con el tópico agua con la que realizamos la "constructed month" en la prensa almeriense.
Como resultados de esta búsqueda obtuvimos un total de 666 noticias para la Vanguardia, 2521 para El Mundo y 1998 para El País, todas ellas relacionadas con el tema del agua.

Como podemos observar en el cuadro III existen dos posibles máximos para los años 2000 y 2004. De estos posibles 


\section{Cuadro III: Frecuencia de noticias para diarios nacionales entre 1992 y 2011}

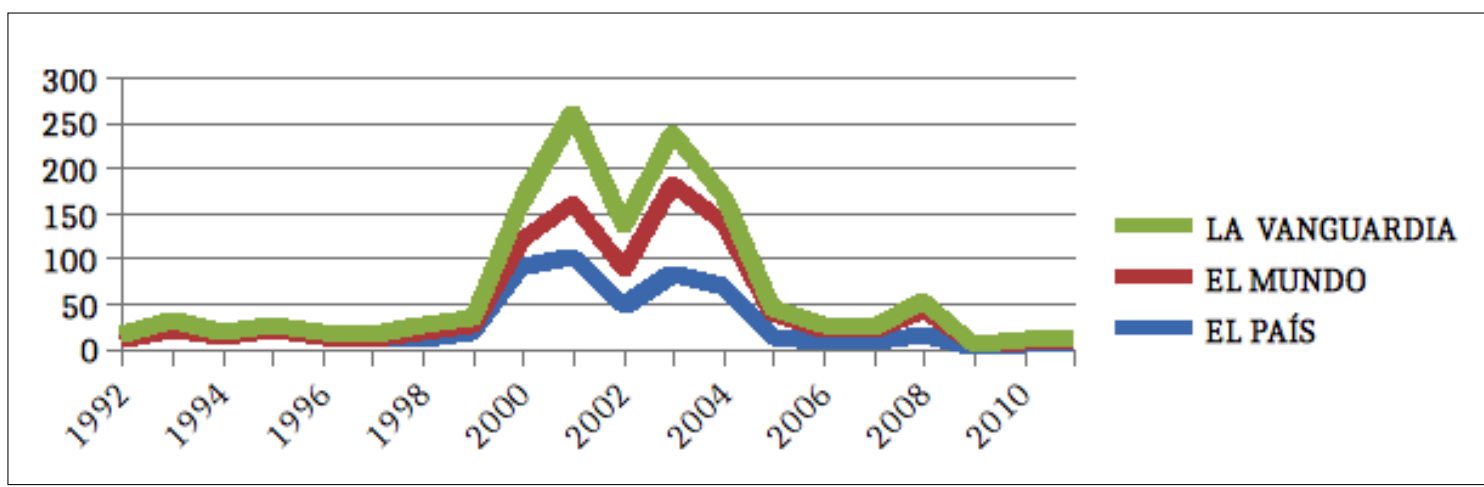

años máximos hemos seleccionado el 2004 porque es el más reciente (Jarman y McClune, 2007) y porque coincide con la convocatoria de elecciones generales en la que la implantación del PHN y su derogación fue utilizada como argumento electoral. Una vez seleccionado el año buscamos el mes con mayor número de artículos relacionados con el tema del agua y para ello comprobamos las publicaciones a lo largo de todo el 2004 en cada periódico nacional.

Podemos observar en el cuadro IV que existe el máximo de presencia de noticias en los tres periódicos nacionales es en el mes de marzo, siendo además el primer mes con frecuencias "al alza".

\section{Cuadro IV: Representación de la frecuencia normalizada por meses con respecto al máximo de cada periódico nacional a lo largo del 2004}

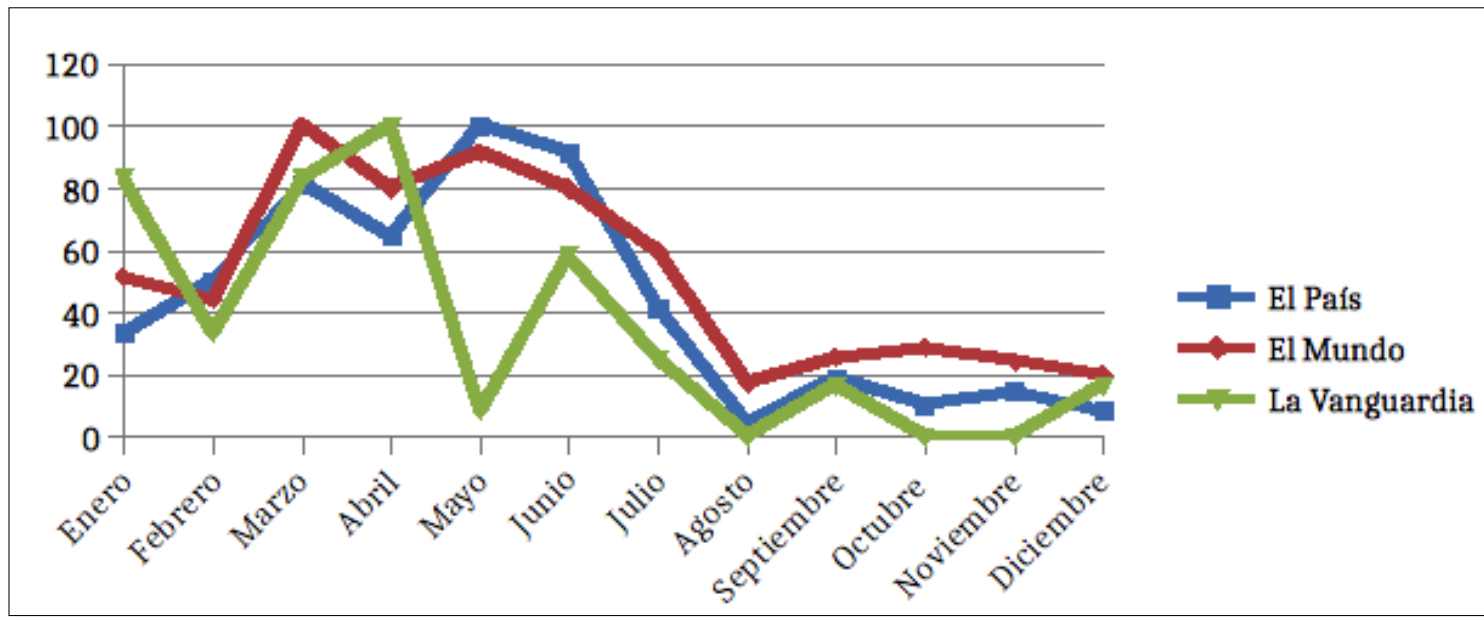




\section{Cuadro V. Titulares de las noticias seleccionadas en el periodo excitado o controvertido}

\begin{tabular}{|c|c|c|}
\hline Título del artículo & Fecha & Nombre del diario \\
\hline $\begin{array}{l}\text { La comisión de Medio Ambiente de la UE desaconseja el } \\
\text { trasvase del Ebro }\end{array}$ & 07/03/2004 & Ideal \\
\hline "Almería no necesita el trasvase del Ebro" & $09 / 03 / 2004$ & Ideal \\
\hline "Hacer un mal uso del agua es ir contra la vida" & $22 / 03 / 2004$ & $\begin{array}{l}\text { Ideal. Suplemento Día } \\
\text { Mundial del Agua }\end{array}$ \\
\hline $\begin{array}{l}\text { "La desalación solar podría tener un hueco entre las distintas } \\
\text { alternativas" }\end{array}$ & $22 / 03 / 2004$ & $\begin{array}{l}\text { Ideal. Suplemento Día } \\
\text { Mundial del Agua }\end{array}$ \\
\hline $\begin{array}{l}\text { Almería carece de agua para garantizar su crecimiento } \\
\text { urbano }\end{array}$ & $28 / 03 / 2004$ & Ideal \\
\hline $\begin{array}{l}\text { La desaladora de Carboneras puede ya ofrecer agua a todo el } \\
\text { campo de Níjar }\end{array}$ & $02 / 03 / 2004$ & La Voz de Almería \\
\hline $\begin{array}{l}\text { La comisión de Medio Ambiente de la UE desaconseja el } \\
\text { trasvase del Ebro }\end{array}$ & 07/03/2004 & La Voz de Almería \\
\hline $\begin{array}{l}\text { Zapatero dice que resolverá el problema del agua sin realizar } \\
\text { trasvases "absurdos". }\end{array}$ & 08/03/2004 & La Voz de Almería \\
\hline El Ebro vierte al mar el equivalente a ocho trasvases & $10 / 03 / 2004$ & La Voz de Almería \\
\hline $\begin{array}{l}\text { El gobierno de Aragón recurre varias obras del trasvase del } \\
\text { Ebro }\end{array}$ & $11 / 03 / 2004$ & La Voz de Almería \\
\hline $\begin{array}{l}\text { La UE examinará una nueva financiación para la gran obra } \\
\text { del PHN }\end{array}$ & $18 / 03 / 2004$ & La Voz de Almería \\
\hline $\begin{array}{l}\text { Denuncian un "mercado negro" del agua en Almería, Murcia } \\
\text { y Alicante }\end{array}$ & $23 / 03 / 2004$ & La Voz de Almería \\
\hline El déficit y cómo lo resolvía el Plan del Agua & $27 / 03 / 2004$ & La Voz de Almería \\
\hline
\end{tabular}

Con este conteo a nivel nacional podemos seleccionar marzo de 2004 como la fecha de mayor controversia nacional y tomarla como periodo de análisis para realizar la "constructed month" en la prensa local, localizando así una muestra representativa de un período de excitación o controvertido con que comparar con el período normal o no controvertido.
La muestra excitada o controvertida está constituida por 69 noticias cuya selección de titulares destacamos en el cuadro V.

\section{Resultados y discusión}

En este apartado desarrollaremos los resultados de presencia obtenidos en las dos muestras de prensa con el fin de determinar cuál es el promedio diario de 
noticias controvertidas del agua en la prensa local y si este varía en distintos períodos de tiempo. Para ello expondremos los resultados en distintos apartados en función de la metodología utilizada.

Resultados de presencia que presentan las noticias según la metodología "constructed week"

Como hemos indicado en el apartado de metodología, al usar la "constructed week" de los 221 artículos relacio- nados con la ciencia y la tecnología, 50 están directamente relacionados con el tema del agua. Esto constituye un $18,5 \%$ del total de noticias científicas encontradas y un promedio diario de 0,47 noticias, resultado de dividir las 50 noticias relacionadas con el tópico agua entre los 105 días (15 semanas completas) muestreados.

Si analizamos la distribución de estas noticias en estado normal sobre agua por periódico y por año obtenemos el cuadro VI.

\section{Cuadro VI: Distribución por años del número de noticias publicadas en el Ideal y La Voz de Almería}

\begin{tabular}{|l|c|c|c|c|c|c|}
\hline & $\mathbf{1 9 9 2}$ & $\mathbf{1 9 9 6}$ & $\mathbf{2 0 0 0}$ & $\mathbf{2 0 0 4}$ & $\mathbf{2 0 0 8}$ & Total \\
\hline La Voz de Almería & 9 & 7 & 6 & 2 & 9 & 33 \\
\hline Ideal & 3 & 0 & 6 & 0 & 8 & 17 \\
\hline Total & 12 & 7 & 12 & 2 & 17 & 50 \\
\hline
\end{tabular}

Para poder comparar nuestros resultados de presencia con un estudio que también esté centrado en un tópico de ciencia y tecnología concreto, utilizaremos el realizado por Jiménez et al. (2010), citado en el cuadro I, donde se investiga otro tópico de especial relevancia en Almería nada controvertido como es la energía solar. Para el caso de la energía solar la frecuencia de noticias es de 43 en 61 días analizados, por lo que se obtiene un promedio diario de 0,7 noticias. Parece por tanto, que las noticias relacionadas con el agua, que como ya hemos comentado presentan un promedio diario de 0,47 , tienen una menor presencia que las de energía solar para los mismos diarios (prensa almeriense) y en periodos similares.

$\mathrm{Al}$ estudiar la distribución por años de las noticias encontramos que existe un mínimo en el 2004 y se inicia un leve ascenso en el 2008 (cuadro VI). Es de destacar que este mínimo en el 2004 de la frecuencia de noticias relacionadas con el agua, se obtenga el mismo año de la derogación del Plan Hidrológico Nacional (2004), incluyendo el Trasvase del Ebro que fue motivo de una fuerte controversia sociocientífica a nivel nacional.

¿Significa esta tendencia que, a pesar de constituir una controversia de 
Cuadro VII: Presencia de noticias, distribuida por semanas completas, en la prensa local en el mes de marzo de 2004

\begin{tabular}{|l|c|c|c|c|}
\hline & La Voz de Almería & Ideal & $\begin{array}{c}\text { Total } \\
\text { noticias }\end{array}$ & $\begin{array}{c}\text { Promedio } \\
\text { diario }\end{array}$ \\
\hline $1^{\text {a }}$ semana (1-7 marzo) & 3 & 12 & 15 & 2,14 \\
\hline $2^{\text {a } \text { semana (8-14 marzo) }}$ & 14 & 5 & 19 & 2,71 \\
\hline $3^{\text {a } \text { semana (15-21 marzo) }}$ & 7 & 10 & 17 & 2,42 \\
\hline $4^{\text {a }}$ semana (22-28 marzo) & 6 & 10 & 16 & 2,28 \\
\hline 29, 30, 31 marzo & 0 & 2 & 2 & 0,66 \\
\hline Total & 30 & 39 & 69 & 2,39 \\
\hline
\end{tabular}

amplio calado nacional, en la prensa almeriense no tuvo repercusión y no se le concedió la suficiente cuota de presencia? Para dar respuesta a esta pregunta necesitamos buscar más información sobre la época de máxima presencia de noticias sobre el aguay, para ello, utilizamos las metodologías "noticia testigo" y "constructed month".

Resultados de presencia que presentan las noticias según la metodología "noticia testigo" y "constructed month"

Como señalamos en el apartado de metodología, en este caso tomamos el mes de marzo de 2004 para obtener más información sobre la presencia del tema del agua en la prensa almeriense.

$\mathrm{Al}$ ampliar la búsqueda de noticias relacionadas con el agua en la prensa local obtuvimos 39 noticias para El Ideal y 30 para La Voz de Almería, es decir, un total es de 69 noticias, con un promedio diario de 2,39 noticias a lo largo del mes de marzo de 2004.
Hemos de señalar que el promedio para los días 29, 30,31 de marzo no participa del promedio total que ha sido calculado utilizando los promedios diarios en las semanas completas.

En el apartado anterior hemos comentado que el promedio de 0,47 obtenido en las quince semanas utilizando la "constructed week" para el tópico agua era muy reducido si lo comparábamos con los datos de presencia del tópico energía solar (0,7 de promedio diario). El promedio para el mes de marzo de $2004(2,39)$ es algo inferior al promedio de todas las noticias científicas (agua, solar, medioambientales, biotecnología, etc.) obtenido por Lapetina (2005) de 2,9 (con suplementos) pero superior al obtenido sin suplementos (1,87, datos extraídos del cuadro I).

Estos datos comparados con otras fechas y con otros tópicos en los mismos periódicos nos da una idea de que en este mes la presencia de noticias sociocientíficas sobre el agua en el mes de marzo de 2004 es elevada en relación a otras noticias científicas o en otros 
periodos en la prensa almeriense (más del triple que la energía solar y ocho veces superior al tema del agua en el periodo elegido al azar de la metodología “constructed week").

Análisis del contenido de las muestras de noticias normal y controvertida

Los titulares expuestos en los cuadros II y V para los periodos normal y controvertido nos permiten una agrupación por similitud para describir las temáticas sobre las que versan y comparar si estas temáticas varían o no dependiendo de la controversia.

Para la muestra del periodo normal hemos detectado como contenidos mayoritarios los relacionados con "gestión del agua" y "gastos en infraestructuras" apareciendo ambos en un $30 \%$ de las noticias. A continuación, los contenidos relacionados con "política" y "calidad del agua" con porcentajes similares del 16 y $14 \%$ respectivamente $\mathrm{y}$, por último, las noticias relacionadas con "déficit hídrico" que aparecen en el $10 \%$ de la muestra normal.

En el caso de la muestra controverti$d a$, se perfilan como contenidos mayoritarios las noticias relacionadas con "política" (42\%) y "gestión del agua" $(33,3 \%)$. A continuación y en porcentajes mucho menores aparecen los contenidos: "déficit hídrico" con un 10,1\%, "gasto en infraestructuras" con un 8,7\% y "calidad del agua" en un 5,8\% de las noticias de la muestra controvertida.

Como podemos observar, las temáticas no varían de un periodo a otro pero al comparar los contenidos de ambas muestras, es de destacar que la temática "política" aumente su presencia del periodo normal al controvertido, sustituyendo a "gastos en infraestructuras" como contenido mayoritario, mientras que "gestión del agua" mantiene resultados similares en ambas muestras. Esta mayor presencia del contenido "política" aparece como consecuencia de haber realizado el muestreo de noticias para la muestra controvertida en periodo pre y post-electoral donde la prensa se hace eco de las campañas de los diferentes partidos.

\section{5. ¿Noticias con diferentes puntos de vista o contraste de noticias consensuadas y controvertidas?}

Como indicamos en párrafos anteriores, la principal utilidad de esta investigación es ayudar a la selección de noticias para el aula de ciencias. En trabajos previos (Díaz y Jiménez, 2012) destacamos que la mayoría de propuestas de uso de controversias sociocientíficas en secundaria planteaban la utilización de varias noticias de prensa con diferentes puntos de vista para generar debate y construcción de argumentaciones a favor o en contra de una determinada controversia en la que suele existir consenso científico. Sin embargo, este planteamiento puede conceder un espacio desproporcionado y colocar en iguales posiciones opiniones que no deberían de tener cabida en el aula generando o reforzando en algunos estudiantes un conocimiento contrario a los fines que se plantean. Estaríamos, por tanto, concediendo repercusión a 
pseudociencias en igualdad de condiciones que a investigación científica, como sucedió recientemente en televisión con la repercusión mediática que se estaba otorgando a los antivacunas en el caso del niño con difteria en Olot $^{2}$.

Solbes (2013b) señala que, en ocasiones, el consenso en la comunidad científica no está tan claro frente a determinadas cuestiones. En estos casos otra manera de abordar las cuestiones sociocientíficas sería presentando a los estudiantes argumentos que usualmente aparecen en los medios de comunicación y pidiéndoles que los pongan en cuestión, como el caso de las centrales nucleares y su prevención en la producción de $\mathrm{CO} 2$, la economicidad de su energía, seguridad, radiación emitida, etc.
Nuestra alternativa a la selección de noticias en función de los pros y contras a una controversia sociocientífica es analizar noticias de igual temática (el agua en la provincia de Almería) elegidas por periodos de normalidad (noticia 1) o controversia (noticia 2).

De las temáticas surgidas en el apartado anterior hemos preferido seleccionar una de cada muestra relacionadas con la gestión del agua porque es uno de los grandes eslóganes de esta tierra de ser eficientes en la gestión del agua pues a pesar de ser un recurso "escaso" en la provincia está muy extendida la idea de la habilidad de las-los agricultores de "aprovechar hasta la última gota".

Por ello, hemos elegido como noticia 1 (Fig. 1) la del 28 de junio del 2008 con

\section{Cuadro VIII. Noticia perteneciente a la muestra normal (noticia 1)}

\section{La eficiente gestión del agua en Almería aterrizará en la Exposición de Zaragoza}

J.E. R. ALMERIA

La provincia de Almeria, ausente hasta el momento de la Bxposicion Universal del Agua que se. celebra en la capital aragonesa. en cuanto a la capacidad de ges. tionar un recurso escaso como es el agua, $y$ la habilidad de los agricultores para utilizar hasta la ultima gota, llegará hasta Zarasoza de la mano de la Cáma. ra de Comercio de Almeria que levará a la 'Cumbre Empresarial Mundial del Asua y el Desarrollo Sostenible' que comenzarí el 1 de julio y se prolongari duran te cuatro jornadas en Zaragoza en el marco de la Bxposición Internacional, la experiencia de Almeria como modelo de efi. ciencia y gestión en la utiliza. ción y aprovechamiento de los recursos hidricos.

Rsta cumbre, está organizada por la Camara de Zangoza, indi. can desde Almeria wsera el instrumento para canalizar las actividades empresariales que intensifiquen la cooperación económica entre Europa y el res: to del mundo, proporcionando a las empresas del sector del agua y el desarrollo sostenible el marco adecuado para incentivar $y$ reforzar su capacidad de gestion en el area internacionale. Entre sus principales objeti. vos, destacan la difusión de los avances e innovaciones en mate ria de agua y sostenibilitad $y$ is puesta a disposiclón de las empresas de una una plataforma que favorezca la realizacion de negocios y la cooperación.

Modelo almeriense La exposicion del modelo almeriense tendrá lugar en la ultima jornada de la Cumbre, el 4 de julio en torno a las 10,00 horas, en el marco de las ponencias dedicadas a la Gestión del Agua y Rxperiencias Regionales. El consejero de Agricultura y Pes ca de la Junta de Andalucia. Martin Soler, será el encargado de presentar la experiencia de la provincia en el transcurso de la ponencia titulada. 'Almeris: un modelo de eficiencia en la gesttón y uso del agua'

${ }^{2}$ http://politica.elpais.com/politica/2015/07/01/actualidad/1435765839_294568.html 
titular "La eficiente gestión del agua en Almería aterrizará en Zaragoza" pues en el texto se hace referencia al modelo de eficiencia, sostenibilidad en la gestión, en la utilización y en el aprovechamiento de los recursos hídricos.

La noticia 2 (Fig. 2), data del 24 de marzo de 2004 y su titular es "Denuncian un "mercado negro" del agua en Almería, Murcia y Alicante”, procedente de la muestra controvertida, hace alusión a la dudosa gestión de recursos hídricos en el Levante español mediante un informe de la Fiscalía de Murcia donde se pone de manifiesto la detracción ilegal de agua en esa comunidad y en las provincias limítrofes lo que permitirá poner en conflicto la eficiencia destacada en la noticia 1.
Las diferencias de enfoque entre ambas noticias deben ser trabajadas en el aula, nuestra propuesta es plantear preguntas en pequeños grupos con el objetivo de analizar ambas noticias relacionadas con el mismo tema (agua) y trabajar tanto la argumentación (Erduran y Jiménez-Aleixandre, 2008) como la comprensión del contenido (Oliveras, Márquez y Sanmartí, 2013) de la ciencia como actividad humana y sus relaciones con la sociedad y el medio ambiente con el fin último de dotarles de estrategias con las que favorecer la fundamentación en la toma de decisiones. Para ello, planteamos una batería de preguntas que pueden realizarse y que, seguro, los docentes que las utilicen adaptarán o ampliarán:

\section{Cuadro IX. Noticia perteneciente a la muestra controvertida (noticia 2)}

\section{REGANTES \\ Denuncian un "mercado negro" del agua en Almería, Murcia y Alicante}

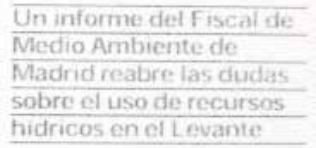

SIMON Rui:

Elfiscal de Medio Ambiente de Ma drid, Emilio Valcrio, ha remitido un informe a la Fiscalia de Murcia en ef que denuncia la existencia de una trama empresarial dedicada la detracción ilegal de agua en esa comunidad. amparada por altos grafica del Segura (CHS)

gráfica del Segura (CHS)
Los diarios "La Opinión de Murcia $y^{*}$ "EI Pais" se hacian eco ayer cia" y "EI Pais" se hacian eco ayer
de esta denuncia. El rotativo murciano aseguraba que el supuesto robo de agua en esa región "se habria repartido también en provincias limitrofes". En el informe se acusa a los responsables "de $22 \mathrm{~cm}$. presas, sociedades agricolas y comunidades de regantes de Murcia.

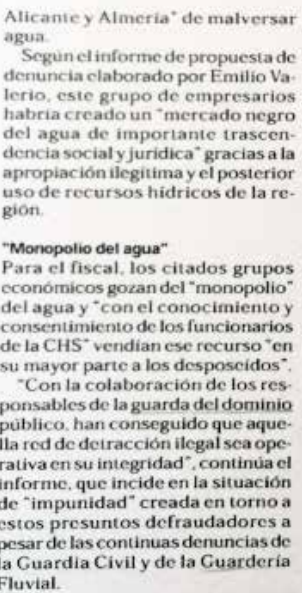

Entre los "denunciados", se encuentra el presidente de ta CHS. Juan Cánovas; el comisario de Aguas del organismo, José Aleman. asícomo otros dos altos cargos de mismo y cl empresario Mariano Ro ca Merono, entre otros administra. dores y socios -en su mayoría de identidad desconocida-del conglomerado de empresas que es denunciado en el 90 por ciento de los 54 puestos tramitados

El fiscal matiza que "de lo investigado no resulta evidencia probatoria en cuanto a una hipotética res. ponsabilidad funcionarial en sede ponsabion funcionarios en sede penal de los funcionarios o autoridades delMinis derio de Medio Am. biente en esta capital (Madrid) en cuanto a los hechos acreditados" Por su parte, el presidente de la Region de Murcia, Ramón Luis Valcárcel, afirmo ayer que "no existen mercados negros del agua" en esta Comunidad y que el caso del supuesto robo de recursos hidricos en el rio Segura, cuyas diligencias se han trasladado del Tribunal Supe rior de Justicia de Madrid al de Mur cia, esta "perdiendo fuerza"
Los presidentes de Valencia y Murcia insisten en pedir el trasvase

Los presidentes de la Comunidad Valenciana, Francisco Camps, y de la Region de Murcia. Ramon Luis Valcarcel, afirmaron ayer, tras una reunion mantenida en el Palau de la Generalitat, que defenderan is ejecucion del trasvase del Ebro previsto en el Plan Hidrologico previsto en el Plan Hidrologico Ne "lealtad" institucional ycti "dealtad" institucional y "dialogo",

" firmeza". pero la comunidades, que abordaron en la comunidades, que abordaron en la coordinar esfruerzos en defensa de coordinar estuerzos en defensa de la realizacion del PHN aprobado por el PP, coincidieron en senalar que el trasvase del Ebro es el solucion al deficit hidrico y en realizar una llamada a la "cooperacion" y al "consenso". 
¿Cuál/es son las ideas principales de cada noticia? Entresacad palabras clave

¿Qué interés/es puede tener quien escribe cada noticia?

¿A quién/es van dirigidas cada noticia? (agricultores-regantes, políticos, votantes, investigadores, etc.)

¿Qué presentan en común las dos noticias y qué aparece de forma diferente?

Una de las noticias está sacada de un periodo de controversia social en relación al tema del agua, ¿cuál crees que puede ser?, ¿en qué te basas para elegir esa como controvertida?

¿Hay distintos intereses en la gestión del agua? ¿Cuáles se entresacan de las noticias?

Si hay un uso eficiente del agua en Almería ¿por qué hay "tráfico" de agua?, ¿a qué crees es debido?

¿No administramos bien el agua?, ¿A qué crees que es debido?

¿Crees que podemos hacer algo al respecto o es una decisión que sólo corresponde a agricultores y políticos?

Propón medidas para gestionar el agua señalando quién debería de realizar cada una de las medias

La familiaridad del tema del agua con el alumnado nos permite que este tema suscite interés y sean participativos, permitiendo además la realización de los debates sin haber asignado roles previamente.

\section{Conclusiones}

Este trabajo pretende contribuir a facilitar el uso de la prensa y más con- cretamente, de noticias con controversias sociocientíficas, en el aula de ciencias por parte del profesorado puesto que sabemos que su uso es prácticamente ausente en la enseñanza reglada a pesar de la gran variedad de posibilidades que presenta (Gadea, Vilches y Gil, 2009). Para ello, hemos estudiado la presencia de una temática local controvertida, el agua en Almería y creemos que puede ser aplicable a otros tópicos que generen controversia en otras localidades.

Al estudiar si varía el número promedio de noticias (presencia) de una determinada temática (agua) en función del periodo seleccionado (normal o controvertido) y la doble metodología utilizada para seleccionar las noticias en ambos periodos ("constructed week" a lo largo de muchos años y "noticia testigo" en un solo mes), estamos ofertando una herramienta útil a los docentes interesados en utilizar prensa y controversias sociocientíficas con la que seleccionar noticias en modo consenso o controversia para su análisis y discusión con el alumnado.

$\mathrm{Al}$ medir la presencia de un tema controvertido en la prensa local, el agua, hemos obtenido mediante catas semanales para un periodo largo en el tiempo (1992-2008) una presencia escasa de noticiaspara la muestra en estado normalsobre el tema del agua en Almería con un promedio de 0,47 artículos diarios y una abundante presencia durante el período excitado o controvertido con un promedio de 2,39 artículos diarios, aunque hemos de señalar que para esta muestra controvertida (marzo de 2004) 
el debate sobre el agua y el PHN estaban en su máximo esplendor. Por tanto, podemos afirmar que, en fechas señaladas en las que el tema del agua aparece de manera importante a nivel nacional, también lo hace a nivel local, mostrándose como controversia sociocientífica en ambos espacios geográficos al mismo tiempo.

A la vista de nuestros resultados, podemos concluir que aunque el tema del agua está a menudo presente en la prensa almeriense, tanto en periodo normal como en controvertido, sin embargo su presencia en la prensa almeriense es representativa cuando se produce una controversia. Estas presencias distintas en ambos periodos y el diferente tratamiento (consensuado o controvertido) nos ha permitido plantear una propuesta sencilla de análisis de controversia de manera que en otros temas conflictivos (vacunas, homeopatías) nos centremos en el contenido evitando conceder excesiva importancia a opiniones que no están fundamentadas en investigación científico-médica alguna.

Por último, hemos incluido unas sugerencias para trabajar noticias controvertidas en el aula que creemos que pueden ser de utilidad para el profesorado y que pretendemos implementar en un futuro y analizar en próximos trabajos.

\section{Referencias bibliográficas}

BAUER, M., DURANT, J., RAGNARSODOTTIR, A. Y RUDOLPHSDOTTIR, A. (1995). Science and technol- ogy in the British press, 1946-1990. London: The Science Museum.

BLANCO, A. (2004). Relaciones entre la educación científica y la divulgación de la ciencia. Revista Eureka sobre Enseñanza y Divulgación de las Ciencias, 1(2), 70-86.

COUSO, D., JIMÉNEZ, M. P., LÓPEZRUÍZ, J., MANS, C., RODRÍGUEZ, C., RODRÍGUEZ, J. M. y SANMARTÍ, N. (2011). Informe ENCIENDE. Enseñanza de las Ciencias en la Didáctica escolar para edades tempranas en España. Madrid: Rubes. Recuperado el 26/08/2013 de http://www.cosce.org/pdf/Informe ENCIENDE.pdf.

DÍAZ, N. (2013). Determinación de una controversia socio-científica a nivel local. El caso del agua como recurso natural en la prensa almeriense. Tesis doctoral, Universidad de Almería.

DÍAZ, N. y JIMÉNEZ, M. R. (2012). Las controversias sociocientíficas: temáticas e importancia para la educación científica. Revista Eureka sobre Enseñanza y Divulgación de las Ciencias, 9 (1), 54-70. DOI: 10498/14624.

DIMOPOULOS, K. y KOULAIDIS, V. (2003). Science and Technology. Education and Citizenship: The potential role of the press. Science Education, 87, 241-256.

DIMOPOULOS, K. y KOULAIDIS, V. (2002). The socio-epistemic constitution of science and technology in the 
Greek press: an analysis of its presentation. Public Understanding of Science, 11, 225-241.

DOMENECH, A.M. (2014). L'ús de les controvèrsies sociocientífiques per promoure la competència científica a l'educació secundària: el cas de la medicalització $i$ el TDA-H (Tesis Doctoral) Recuperada de http://www. tdx.cat/handle/10803/283516

ECOBARÓMETRO (2004). Web de la Consejería de Medio Ambiente y Ordenación del Territorio. Disponible en http://goo.gl/1sv6Q8.

ECOBARÓMETRO (2010). Web de la Consejería de Medio Ambiente y Ordenación del Territorio. Disponible en http://goo.gl/dGorNi.

ERDURAN, S., Y JIMÉNEZ-ALEIXANDRE, M. P. (2008). Argumentation in science education. Perspectives.

GADEA, I., VILCHES, A. y GIL, D. (2009). Posibles usos de las prensa en la educación científica y tecnológica. Didáctica de las Ciencias Experimentales y Sociales, 23, 153-169.

HANSEN, A., COTTLE, S., NEGRINE, R. y NEWBOLD, C. (1998). Mass communication research methods. London: Macmillan.

JARMAN, R. y MCCLUNE, B. (2007). Developing Scientific Literacy. Using News Media in the Classroom. New York: McGraw-Hill.

JIMÉNEZ, M. R.; HERNÁNDEZ, L. y LAPETINA J. (2010). Dificultades y propuestas para utilizar las noticias científicas de la prensa en el aula de ciencias. Revista Eureka sobre Enseñanza y Divulgación de las Ciencias, 7 (1), 107-126. DOI: 10498/9866

LAPETINA, J. (2005). La divulgación científica a través de la prensa escrita de Almería (1992 - 2004). Memoria no publicada para la obtención del DEA. Universidad de Granada. Granada.

METCALFE, J. y GASCOIGNE, T. (1995). Science journalism in Australia. Public Understanding of Science, 4, 411-428.

OLIVERAS, B., MÁRQUEZ, C., Y SANMARTÍ, N. (2013). The use of newspaper articles as a tool to develop critical thinking in science classes. International Journal of Science Education, 35(6), 885-905. http://dx. doi.org/10.1080/09500693.2011.586 736

PEDRINACI, E., CAAMAÑO, A., CAÑAL, P. y DE PRO, A. (2012). 11 ideas clave. El desarrollo de la competencia científica. Barcelona: Graó.

PELLENCHIA, M. G. (1997). Trends in science coverage: A content analysis of three US newspapers. Public Understanding of Science, 6, 49-68.

PUIG, B., BRAVO, B., y JIMÉNEZALEIXANDRE, M. P. (2012). Argumentación en el aula: Dos unidades didácticas. Santiago de Compostela: Danú. Proyecto S-TEAM. [hay versiones en gallego, castellano e inglés].

SOLBES, J. (2013 a). Contribución de las cuestiones sociocientíficas al desarrollo del pensamiento crítico 
(I): Ejemplos. Revista Eureka sobre Enseñanza y Divulgación de las Ciencias, 10(1), 1-10. DOI: 10498/14993

SOLBES, J. (2013b). Contribución de las cuestiones sociocientíficas al desarrollo del pensamiento crítico (II): Ejemplos. Revista Eureka sobre Enseñanza y Divulgación de las Ciencias, 10(2), 171-181. DOI: 10498/15113
SOLBES, J. Y TORRES, N. (2012). Análisis de las competencias del pensamiento crítico desde el aborde de las cuestiones sociocientíficas: un estudio en el ámbito universitario. Didáctica de las Ciencias Experimentales y Sociales, 26, 247-269. DOI: 10.7203/ DCES.26.1928 
